\title{
EWSR1-CREB1 Fusion Protein Expression
}

National Cancer Institute

\section{Source}

National Cancer Institute. EWSR1-CREB1 Fusion Protein Expression. NCI Thesaurus. Code $\mathrm{C121772.}$

Expression of fusion protein EWSR1-CREB1 resulting from a $t(2 ; 22)(q 33 ; q 12)$. It is present in the vast majority of angiomatoid fibrous histiocytoma cases. 